\title{
A new proposal for Galactic dark matter: Effect of $f(T)$ gravity
}

\author{
Farook Rahaman* \\ Department of Mathematics, Jadavpur University, Kolkata 700 032, West Bengal, India \\ Ritabrata Biswa: \\ Department of Physics, IISc, Bangalore, India \\ Hafiza Ismat Fatimd \\ Department of Mathematics, Govt. Degree college (W) Warburton, Nankana Sahib, Pakistan \\ Nasarul Islans \\ Department of Mathematics, Danga High Madrasah, Kolkata 700 103, West Bengal, India
}

(Dated: September 25, 2018)

\begin{abstract}
It is still a challenging problem to the theoretical physicists to know the exact nature of the galactic dark matter which causes the galactic rotational velocity to be more or less a constant. We have proposed the dark matter as an effect of $\mathrm{f}(\mathrm{T})$ gravity. Assuming the flat rotation curves as input we have shown that $\mathrm{f}(\mathrm{T})$ gravity can explain galactic dynamics. Here, we do not have to introduce dark matter. Spacetime metric inspired by $\mathrm{f}(\mathrm{T})$ gravity describes the region up to which the tangential velocity of the test particle is constant. This inherent property appears to be enough to produce stable circular orbits as well as attractive gravity.

PACS numbers: $04.40 . \mathrm{Nr}, 04.20 . \mathrm{Jb}, 04.20 . \mathrm{Dw}$
\end{abstract}

\section{INTRODUCTION}

The sources that are responsible for the rotation curves of neutral hydrogen clouds in the outer regions of galaxy remain an active area of research in recent time. The observed luminous matter content of the galaxies does not follow this behavior. In other words, either Newtonian gravity and general theory of gravity fail to explain this phenomena or there exists some non luminous matter hidden in the galaxy. About 80 years ago, Zwicky [1] confirmed the existence of non luminous matter, so called dark matter, by comparing the virial mass and luminous mass of the galactic clusters. The nature of this non luminous matter contained in the galaxy is still unknown as this matter does not follow particle standard model. In spite of several analytic models are proposed time to time. For a summary of some alternative theories such as noncommutative-geometry background, scalar-tensor theory, brane-world models, see the references [2]-[10]. Some authors have considered quintessential matter, perfect fluid type and global monopoles as a candidate for galactic halo matter [11]-[13].

Teleparallel equivalent of general relativity (TEGR) received a great attention as alternative theory of gravity. In this new theory, it is assumed that the manifold contains, in addition to curvature, a quantity called torsion. This theory is known as $f(T)$ theory. In analogous to the

\footnotetext{
*Electronic address: rahaman@iucaa.ernet.in

${ }^{\dagger}$ Electronic address: ritabrata@physics.iisc.ernet.in

‡Electronic address: hafizaismatfatima@yahoo.com

$\S$ Electronic address: nasaiitk@gmail.com
}

$f(R)$ theory, $f(T)$ theory is constructed with a generalized Lagrangian that deviates from Einstein gravity by a function $f(T)$, where $T$ is the so-called torsion scalar [14]-[15]. Recently, some authors [16] show that the $f(T)$ gravity theory is extensively used to explain the accelerated state of the Universe without introducing the dark energy.

Deliduman and Yapiskan 24] have observed the constraint on the neutron solution in $f(T)$ theory of gravity and concluded that the solution is possible only for a linear function of torsion. Cai et al. [25] have discussed the matter bounce cosmology with scalar and tensor modes of cosmological perturbations. Böhmer et al. 17] have investigated the existence of relativistic stars taking different approaches in this theory and have constructed static perfect fluid solutions.

In this paper, we propose the source of the galactic halo as an effect of $f(T)$ gravity in addition to the normal matter as luminous matter. All the astrophysical observations made in last one and half decades require the modification of either side of the Einstein gravity for their theoretical explanations. Galactic halo is popularly explained by the effect of dark matter . However, as yet no convincing argument about the origin of the angular momentum has been suggested. Hoyle [18] first proposed gravitational instability, arising from gravitational coupling with the surrounding matter (tidal interactions), as a possible explanation for galactic rotation. Alternatively, peoples have proposed [19, 20] that the origin of galaxy rotation could be due to primordial turbulence/vorticity. However, vortical modes decay with time and, therefore, a significant vorticity at the time of galaxy formation would typically require an unrealistic magnitude of vorticity in the early universe. At present, 
it is widely believed that the hierarchical clustering of cold dark matter [21] is the origin of structures in the universe. Consequently, the angular momentum of dark matter halos and eventually the rotation of galaxies is thought to be produced by gravitational tidal torque [22]. But inclusion of Dark matter means the modification of the energy momentum tensor in Einstein equation. Now in spite of doing that we are free to modify the geometry side of the Einstein equation to support the same observation. It means we can modify our gravity. Here we have chosen the TEGR as a modification of gravity. Now our motive is to replace the dark matter by modified gravity. It is argued that by using modified gravity, one can find gravitational analog of the Lorentz force equation, which is an equation written in the underlying Weitzenbock spacetime [23]. According to this approach, the trajectory of the particle is described in the very same way the Lorentz force describes the trajectory of a charged particle in the presence of an electromagnetic field, with torsion playing the role of force. These all uses made us more interested to find a way to try to interpret the galactic halo as a result of TEGR rather than dark matter.

The outline of the paper is as follows: In the next section, we discuss the galactic rotation curves using a spherical gravitational field. Section III provides the field equations in $f(T)$ theory and their solutions. Section IV discusses the results of the solutions for $\frac{d f}{d T}=1$ and $\frac{d f}{d T} \neq 1$ and gives several observations following the obtained solutions. The last section $\mathrm{V}$ contains concluding remarks about the proposal.

\section{GALACTIC ROTATION CURVES}

The observation of the motion of neutral hydrogen clouds are believed as evidence for the existence of some kind of exotic matter in the galactic halo apart from luminous matter in the galaxies. These hydrogen clouds in the outer region of galaxies are treated as test particles moving in circular orbits due to gravitational effects of the combined matters ( luminous matter as normal matter and non luminous matter as dark matter ) contained in the galaxies. The gravitational field inside the halo is characterized by line element

$$
d s^{2}=-e^{\nu(r)} d t^{2}+e^{\lambda(r)} d r^{2}+r^{2} d \Omega^{2},
$$

where $d \Omega^{2}=d \theta^{2}+\sin ^{2} \theta d \phi^{2}$. We are using here geometrized units in which $G=c=1$.

To study the tangential velocity of such circular orbits, we will derive geodesic equation from the Lagrangian for a test particle travelling on the spacetime (1) which is given by

$$
2 \mathcal{L}=-e^{\nu(r)} \dot{t}^{2}+e^{\lambda(r)} \dot{r}^{2}+r^{2} \dot{\Omega}^{2},
$$

where as usual $d \dot{\Omega}^{2}=d \dot{\theta}^{2}+\sin ^{2} \theta d \dot{\phi}^{2}$. The overdot denotes differentiation with respect to affine parameter $s$.
From (2), the geodesic equation for material particle can be written as

$$
\dot{r}^{2}+V(r)=0
$$

now yields the potential

$$
V(r)=-e^{-\lambda(r)}\left(e^{-\nu(r)} E^{2}-\frac{L^{2}}{r^{2}}-1\right) .
$$

Here the conserved quantities $E$ and $L$, the energy and total momentum, respectively are given by $E=-e^{\nu(r)} \dot{t}$, $L_{\theta}=r^{2} \dot{\theta}$, and $L_{\phi}=r^{2} \sin ^{2} \theta \dot{\phi}$. So the square of the total angular momentum is $L^{2}=L_{\theta}{ }^{2}+\left(L_{\phi} / \sin \theta\right)^{2}$.

Following [2, 3], one can find the tangential velocity of the test particle,

$$
\left(v^{\phi}\right)^{2}=\frac{e^{\nu} L^{2}}{r^{2} E^{2}}=\frac{1}{2} r \nu^{\prime} .
$$

This expression can be integrated to yield the metric coefficient at the distances where the circular velocity remains constant with radius

$$
e^{\nu}=B_{0} r^{l}
$$

where $B_{0}$ is an integration constant and $l=2\left(v^{\phi}\right)^{2}$.

Equivalently, the line element (1) in regions with a flat rotational curve

$$
d s^{2}=-B_{0} r^{l} d t^{2}+e^{\lambda(r)} d r^{2}+r^{2} d \Omega^{2} .
$$

It seems to me the source of galactic matter as an effect of $f(T)$ gravity in addition to the normal matter as luminous matter. Therefore, it is interesting to find spacetime metric of the region of constant tangent velocity.

\section{FIELD EQUATIONS IN $F(T)$ THEORY :}

Similar to $f(R)$ modified theory of gravity, the action of $f(T)$ theory is taken as [14]- 17 ]

$$
S\left[e_{\mu}^{i}, \phi_{A}\right]=\int d^{4} x\left[\frac{1}{16 \pi} f(T)+L_{\text {matter }}\left(\phi_{A}\right)\right]
$$

Here, $e_{\mu}^{i}$ are the tetrad by which we can define any metric as $g_{\mu \nu}=\eta_{i j} e_{\mu}^{i} e_{\nu}^{j}$, where $\eta_{i j}=\operatorname{diag}(-1,1,1,1)$ and $e_{i}^{\mu} e_{\nu}^{i}=\delta_{\nu}^{\mu}, e=\sqrt{-g}=\operatorname{det}\left(e_{\mu}^{i}\right)$.

We used the units $G=c=1$. Here, $\phi_{A}$ are matter fields and $f(T)$ is an arbitrary analytic function of the torsion scalar $T$. The torsion scalar is built from torsion and contorsion as

$$
T=S_{\sigma}^{\mu \nu} T_{\mu \nu}^{\sigma}
$$

where,

$$
T_{\mu \nu}^{\sigma}=\Gamma_{\mu \nu}^{\sigma}-\Gamma_{\nu \mu}^{\sigma}=e_{i}^{\sigma}\left(\partial_{\mu} e_{\nu}^{i}-\partial_{\nu} e_{\mu}^{i}\right)
$$




$$
K_{\sigma}^{\mu \nu}=-\frac{1}{2}\left(T_{\sigma}^{\mu \nu}-T_{\sigma}^{\nu \mu}-T_{\sigma}^{\mu \nu}\right)
$$

are torsion and contorsion respectively with new defined tensor components

$$
S_{\sigma}^{\mu \nu}=\frac{1}{2}\left(K_{\sigma}^{\mu \nu}+\delta_{\sigma}^{\mu} T_{\beta}^{\beta \nu}-\delta_{\sigma}^{\nu} T_{\beta}^{\beta \mu}\right)
$$

Varying the action (8) with respect to the tetrads, one can obtain the field equations of $f(T)$ gravity as

$S_{i}^{\mu \nu} f_{T T} \partial_{\mu} T+e^{-1} \partial_{\mu}\left(e S_{i}^{\mu \nu}\right) f_{T}-T_{\mu i}^{\sigma} S_{\sigma}^{\nu \mu} f_{T}+\frac{1}{4} e_{i}^{\nu} f=4 \pi \Upsilon_{i}^{\nu}$

where,

$$
S_{i}^{\mu \nu}=e_{i}^{\sigma} S_{\sigma}^{\mu \nu}, \quad f_{T}=\frac{\partial f}{\partial T} \quad \text { and } \quad f_{T T}=\frac{\partial^{2} f}{\partial T^{2}}
$$

and $\Upsilon_{i}^{\nu}$, the energy momentum tensor of the anisotropic fluid is given by

$$
\Upsilon_{\nu}^{\mu}=\left(\rho+p_{r}\right) u^{\mu} u_{\nu}-p_{r} g_{\nu}^{\mu}+\left(p_{t}-p_{r}\right) \eta^{\mu} \eta_{\nu}
$$

with

$$
u^{\mu} u_{\mu}=-\eta^{\mu} \eta_{\mu}=1
$$

For our metric (11), we define the tetrad matrix as

$$
e_{\mu}^{i}=\operatorname{diag}\left[\sqrt{B_{0} r^{l}}, e^{\frac{\lambda}{2}}, r, r \sin \theta\right] .
$$

Now, we compute the torsion scalar as

$$
T(r)=-\frac{2 e^{-\lambda}}{r}\left(\frac{l}{r}+\frac{1}{r}\right)
$$

Inserting this and the components of the tensors $S_{i}^{\mu \nu}$ and $T_{i}^{\mu \nu}$ in (13) yields,

$$
\begin{gathered}
4 \pi \rho=\frac{e^{-\lambda}}{r} T^{\prime} f_{T T}-\left[\frac{T}{2}+\frac{1}{2 r^{2}}+\frac{e^{-\lambda}}{2 r}\left(\frac{l}{r}+\lambda^{\prime}\right)\right] f_{T}+\frac{f}{4} \\
4 \pi p_{r}=\left[\frac{T}{2}+\frac{1}{2 r^{2}}\right] f_{T}-\frac{f}{4} \\
4 \pi p_{t}=-\frac{e^{-\lambda}}{2}\left(\frac{l}{2 r}+\frac{1}{r}\right) T^{\prime} f_{T T}+\frac{T}{4} f_{T} \\
-\frac{e^{-\lambda}}{2}\left[\frac{-l}{2 r^{2}}+\left(\frac{l}{4 r}+\frac{1}{2 r}\right)\left(\frac{l}{r}-\lambda^{\prime}\right)\right] f_{T}-\frac{f}{4}
\end{gathered}
$$

According to Böhmer et al [17], one of the possibility of recovering general relativity result is $f_{T T}=0$, though its general relativity form has no meaning in the present context.

\section{SOLUTIONS AND THEIR CONSEQUENCES}

Now we are seeking solutions using some specific forms of $\mathrm{f}(\mathrm{T})$.

$$
\text { A. } f(T)=T
$$

Let us seek the influence of $\mathrm{f}(\mathrm{T})$ gravity by assuming $f_{T}=1$. This assumption immediately yields

$$
f(T)=T
$$

which immediately follows $f_{T T}=0$. For normal matter, we have

$$
p_{r}=m \rho
$$

where, ' $m$ ' is an equation of state parameter.

Now, using equation (19), solving $e^{-\lambda(r)}$ from (20), (16) and (17) we have,

$$
e^{-\lambda(r)}=\left[\frac{1+m}{m+l+1}\right]+D r^{-\left[\frac{m+l+1}{m}\right]}
$$

where, ' $D^{\prime}$ is an integration constant. Then, from equation (15) we can obtain

$T(r)=-\left[\frac{2(1+m)(1+l)}{(m+l+1)}\right] r^{-2}-2 D(l+1) r^{-\left[\frac{3 m+l+1}{m}\right]}$,

$4 \pi \rho(r)=-\left[\frac{D(l+1)}{2 m}\right] r^{-\left[\frac{3 m+l+1}{m}\right]}+\left[\frac{l}{2(m+l+1)}\right] r^{-2}$

and

$$
\begin{aligned}
4 \pi p_{t}=\left[\frac{D(l+2)(m+l+1)}{16 m(1+l)}\right] r^{-\left[\frac{3 m+l+1}{m}\right]} & \\
& \quad-\frac{l^{2}}{8 r^{2}}\left[\left(\frac{1+m}{m+l+1}\right)+D r^{\left.-\left[\frac{m+l+1}{m}\right]\right]}\right.
\end{aligned}
$$

\section{Comments on the solutions :}

(a) Notice that this type of spacetime definitely cannot be asymptotically flat neither can it have the form of a spacetime due to a centrally symmetric black hole. Since this space describes the region up to which tangential velocity is constant, so it has to be joined with exterior region with other types of spacetime, may be with Schwarzschild spacetime. Thus, in principle, one can obtain the value of $D$ with the junction conditions. However, it is yet to discover the galactic boundary.

(b) The remarkable effect due to $f(T)$ gravity is the presence of first term in the expression of energy density . According to Newtonian theory which is indistinguishable from general relativity in very weak field, to maintain circular orbits of the neutral hydrogen clouds with constant velocity, the centrifugal acceleration $\frac{\left(v^{\phi}\right)^{2}}{r}$ should be equal to the gravitational attraction $\frac{G M(r)}{r^{2}}$ of the total mass $M(r)$ contained within radius $r$. This yields the Newtonian mass $M(r)$ is increasing linearly with $r$. In other words, $\rho_{\text {Newton }} \propto \frac{1}{r^{2}}$. The first term in 
the expression of energy density due to the effect of $f(T)$ gravity. This contribution will vanish if $D$ vanishes.

(c) Now, we try to recognize $D$. Our metric (7) will take the following in the limit $l \rightarrow 0$ as $d s^{2}=$ $-d t^{2}+\frac{1}{1+D r^{-\frac{m+1}{m}}} d r^{2}+r^{2} d \Omega^{2}$. The standard FLRW metric is $d s^{2}=-d t^{2}+\frac{1}{1-k r^{2}} d r^{2}+r^{2} d \Omega^{2}$. If one considers, $m=-\frac{1}{3}$, then, one can lead identification that $D=-k$, in other words, $D$ is recognized as the spatial curvature (with a negative sign). Thus, one may assume our obtained spacetime metric of the galactic halo is induced by the space-time embedded in a static FLRW metric.
It is known that spatially curved FLRW universe would be static and stable if the universe is supported by the fluid with equation of state $\rho=-3 p$. The dynamicity of FLRW metric is absent here, as we are working on a local problem like flat rotation curve which fixes the scale factor $R(t)$ to be constant. The fluid following equation of state $\rho=-3 p$ is not exotic as it follows null and weak energy condition. Hence, we don't need to consider dark matter as exotic matter. It is nothing but an effect of $f(T)$ theory. Now, we calculate Ricci scalar $\left(R_{c}\right)$ for the derived spacetime which is given by,

$$
R_{c}=\frac{D\left[\left(\frac{m+l+1}{m}\right)^{2}(4+l)\right]-\left[\left(\frac{m+l+1}{m}\right) D+\left(\frac{m+1}{m}\right) r^{\left(\frac{m+l+1}{m}\right)}\right]\left(l^{2}+2 l+4\right)+4\left(\frac{m+l+1}{m}\right) r^{\left(\frac{m+l+1}{m}\right)}}{2\left[\left(\frac{m+l+1}{m}\right) r^{2+\left(\frac{m+l+1}{m}\right)}\right]}
$$

As $l \rightarrow 0$ and $m=-\frac{1}{3}, R_{c}=-6 D$, once again suggesting that $D$ is the spatial curvature.

However, in general $l \neq 0$ and hence the above interpretation is not universally truth.

(d) If we write $e^{\lambda}$ in the standard Schwarzschild form

$$
e^{\lambda}=\left[1-\frac{2 \mathcal{M}(r)}{r}\right]^{-1}
$$

then our model reveals that the proper radial length is larger than the Euclidean length because $r>2 \mathcal{M}(r)$. This is a very crucial condition for a valid metric as this condition keeps safe the signature. For example, if we put $D=0$ in our metric function (21), we get,

$$
e^{\lambda}=\frac{1+m+l}{1+m}=1+\frac{l}{1+m}>1
$$

This implies $D=0$ is one of the possible values which fulfils the essential requirement.

(e) After performing the radial rescaling

$$
r=\sqrt{\frac{1+m}{1+m+l}} R,
$$

one can rewrite the metric (7) for the solution (22) with $D=0$ as

$d s^{2}=-B_{0}^{\prime} R^{l} d t^{2}+d R^{2}+\left[\frac{1+m}{1+m+l}\right] R^{2}\left(d \theta^{2}+\sin ^{\theta} d \phi^{2}\right)$

where, $B_{0}^{\prime}=B_{0}\left(\sqrt{\frac{1+m}{1+m+l}}\right)^{l}$.

This shows an angle of surplus (analogous to the angle of deficit ) in the surface area given by

$$
S_{1}=4 \pi R^{2}\left[\frac{1+m}{1+m+l}\right]=\left[\frac{1+m}{1+m+2\left(v^{\phi}\right)^{2}}\right] 4 \pi R^{2}
$$

One can observe that if the rotational velocity of the probe particles wee photons so that $v^{\phi} \rightarrow 1$, the surface would remain finite, however reduced to the factor $\left[\frac{1+m}{3+m}\right]$ of the spherical surface area $S_{2}=4 \pi R^{2}$. However, for a typical galaxy, the rotational velocity is $v^{\phi} \approx 10^{-3}$ and in this case the difference of two surface areas

$$
S_{2}-S_{1}=4 \pi R^{2}\left[\frac{2\left(v^{\phi}\right)^{2}}{1+m+2\left(v^{\phi}\right)^{2}}\right]
$$

One can note that for increasing the value of equation of state parameter, this difference decreases. Thus rate of the difference in units of flat surface area grows as $\approx 10^{-6}$. Definitely, nowadays, this measurement is sufficient to examine the actual behavior of the motion of stars in a galaxy.

(f) It can be verified that $\rho>0, \rho+p_{r}>0, \rho+p_{r}+2 p_{t}>$ 0 . This means halo contains non exotic matter as matter satisfies all energy conditions. Therefore, we expect an attractive halo. Following Lynden-Bell et al's [26] prescription we ratify it by calculating the total gravitational energy $E_{G}$ which is given by

$$
E_{G}=M-E_{M}=4 \pi \int_{r_{1}}^{r_{2}}\left[1-\sqrt{e^{\lambda(r)}}\right] \rho r^{2} d r
$$

Note that, since $\rho>0,1-\sqrt{e^{\lambda(r)}}<0$ and $r_{2}>r_{1}$, therefore, $E_{G}<0$, showing that gravity in the halo is indeed attractive.

Also, one can confirm that the effect is attractive by 
studying the geodesic equation

$$
\frac{d^{2} x^{\alpha}}{d \tau^{2}}+\Gamma_{\alpha}^{\mu \gamma} \frac{d x^{\mu}}{d \tau} \frac{d x^{\gamma}}{d \tau}=0
$$

for a test particle that has placed at some radius $r_{0}$. This yields the radial equation

$$
\frac{d^{2} r}{d \tau^{2}}=-\frac{1}{2} e^{-\lambda}\left[\frac{d}{d r} e^{\lambda}\left(\frac{d r}{d \tau}\right)^{2}+\frac{d}{d r} e^{\nu}\left(\frac{d t}{d \tau}\right)^{2}\right]
$$

. As long as $\frac{d r}{d \tau}=0$, we get

$$
\frac{d^{2} r}{d \tau^{2}}=-\frac{1}{2} e^{-\lambda} B_{0} l r^{l-1}\left(\frac{d t}{d \tau}\right)^{2}<0 .
$$

We conclude that objects are attracted toward the center. This result agrees with the observational evidence i.e., gravity on the galactic scale is attractive ( clustering , structure formation etc ).

(g) To check stability of circular orbits, let us assume a test particle with four velocity $U^{\alpha}=\frac{d x^{\sigma}}{d \tau}$ moving in the region of spacetime given in (7). Assuming $\theta=\pi / 2$, the equation $g_{\nu \sigma} U^{\nu} U^{\sigma}=-m_{0}^{2}$ yields

$$
\left(\frac{d r}{d \tau}\right)^{2}=E^{2}+V(r)
$$

with

$$
V(r)=-\left[E^{2}\left(1-\frac{r^{-l} e^{-\lambda}}{B_{0}}\right)+e^{-\lambda}\left(1+\frac{L^{2}}{r^{2}}\right)\right] .
$$

where, $E=\frac{U_{0}}{m_{0}} \quad$ and $L=\frac{U_{3}}{m_{0}}$ are two conserved quantities, namely relativistic energy (E) and angular momentum (L) per unit rest mass of the test particle respectively. If the circular orbits are defined by $r=R$, then $\frac{d R_{0}}{d \tau}=0$ and, additionally, $\left.\frac{d V}{d r}\right|_{r=R}=0$. Above two conditions result

$$
L= \pm \sqrt{\frac{l}{2-l}} R
$$

and, using $L$ in $V(R)=-E^{2}$, we get

$$
E= \pm \sqrt{\frac{2 B_{0}}{2-l}} R^{\frac{l}{2}}
$$

The orbits will be stable if $\left.\frac{d^{2} V}{d r^{2}}\right|_{r=R}<0$ and unstable if $\left.\frac{d^{2} V}{d r^{2}}\right|_{r=R}>0$.

By putting the expressions for $L$ and $E$ in $\left.\frac{d^{2} V}{d r^{2}}\right|_{r=R}$ and then by using the solution (14), we get,

$$
\left.\frac{d^{2} V}{d r^{2}}\right|_{r=R}=-\left[\frac{2 l(1+m)}{(1+m+l) R^{2}}+\frac{D l(3 m+l+1)}{m(2-l)(1+l)} R^{\frac{-(3 m+l-1)}{m}}\right]
$$

One may note that, for $D=0$ as well as for $D \neq 0$, $\left.\frac{d^{2} V}{d r^{2}}\right|_{r=R}<0$. So the circular orbits are always stable.

$$
\text { B. } f(T)=\alpha T+T_{0}
$$

In the previous section, we have investigated the influence of $\mathrm{f}(\mathrm{T})$ gravity with $f_{T}=1$. Now, we will assume the case $f_{T} \neq 1$ and consider

$$
f(T)=\alpha T+T_{0}
$$

where $T_{0}$ and $\alpha$ are constants.

Again, one can solve for $e^{-\lambda(r)}$ from the field equations (16) - (18) by using equation(30) as

$$
e^{-\lambda(r)}=\frac{1+m}{1+m+l}+D_{2} r^{-\frac{l+m+1}{m}}-\frac{T_{0}(1+m)}{2 \alpha(3 m+l+1)} r^{2}
$$

$D_{2}$ being constant of integration.

Accordingly, we have,

$$
\begin{array}{r}
T(r)=\frac{T_{0}(1+m)(l+1)}{\alpha(3 m+l+1)}-\frac{2(1+m)(1+l)}{1+m+l} r^{-2} \\
-2 D_{2}(l+1) r^{-\frac{l+3 m+1}{m}}
\end{array}
$$

$$
\begin{array}{r}
4 \pi \rho=\frac{-\alpha}{4}\left[\frac{T_{0}(1+m)(l+1)}{\alpha(3 m+l+1)}-\frac{2(1+m)(1+l)}{1+m+l} r^{-2}-2 D_{2}(l+1) r^{-\frac{l+3 m+1}{m}}\right]-\frac{\alpha r^{-2}}{2}-\frac{l \alpha(1+m)}{2(l+m+1)} r^{-2} \\
-\frac{\alpha}{2}\left(\frac{l+m(l+1)+1}{m}\right) D_{2} r^{-\frac{3 m+l+1}{m}}+\frac{T_{0}}{4}\left(\frac{(l+1) m+2 l}{3 m+l+1}\right)
\end{array}
$$


$16 \pi p_{r}=\alpha\left[\frac{T_{0}(1+m)(l+1)}{\alpha(3 m+l+1)}-\frac{2(1+m)(1+l)}{1+m+l} r^{-2}-2 D_{2}(l+1) r^{-\frac{l+3 m+1}{m}}\right]+2 \alpha r^{-2}-T_{0}$

$$
\begin{array}{r}
16 \pi p_{t}=-T_{0}-\frac{\alpha l^{2}}{2}\left[\left(\frac{1+m}{1+m+l}\right) r^{-2}+D_{2} r^{-\frac{l+3 m+1}{m}}-\frac{T_{0}(1+m)}{2 \alpha(5 m+l+1)}\right] \\
+\frac{\alpha(l+2)}{2}\left[\frac{D_{2}(l+m+1)}{m} r^{-\frac{3 m+l+1}{m}}+\frac{T_{0}(1+m)}{\alpha(3 m+l+1)}\right]
\end{array}
$$

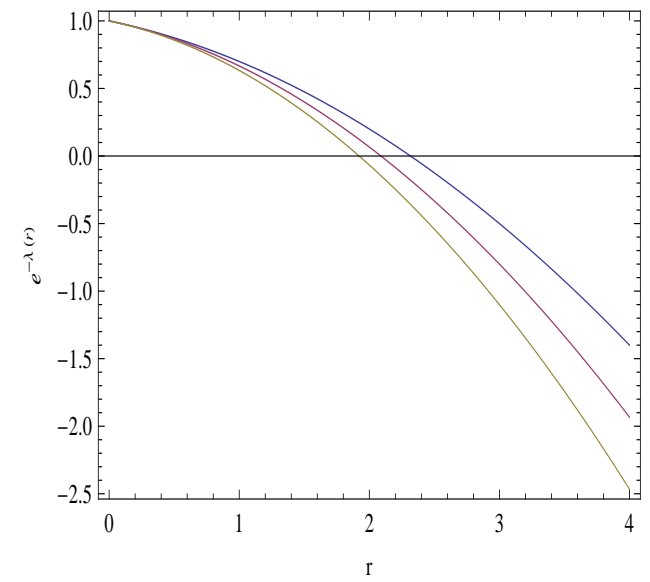

FIG. 1: The singularity occurs where $e^{-\lambda}$ cuts $\mathrm{r}$ axis.

\section{Several observations follow from the solutions:}

(a) Point to be noticed is that the solution indicates almost the same kind of space time denoted by the Case $f(T)=T$ solution.

(b) We can check in the energy density's expression, the second term in first bracket only the term not containing any information from $f(T)$ gravity. Even this guarantees the fact $\rho_{\text {Newton }} \propto \frac{1}{r^{2}}$. Making $\alpha=1$ and $D_{2}=T_{0}=0$ together, we can go back to the Newtonian solution.

(c) While physically interpreting $D_{2}$, we should check for the metric (7) for $l \rightarrow 0$ and comparing with FLRW metric, we have for $m=-\frac{1}{3}, \frac{T_{0}}{2 \alpha}+D_{2}=-\kappa, \kappa$ being the scalar curvature. Interestingly, for $D_{2}=-\frac{T_{0}}{2 \alpha}$, the spacetime would be flat. It is to be noted that we are at the Strong energy condition boundary with such an $m$. (d) It is quite obvious that we will not be able to find exact form of black hole singularity with such a result as trying to make the $e^{-\lambda(r)}=0$, will lead us to a transcendental equation. If we plot $e^{-\lambda(r)}$ vs $r$ to find its zeroes, then we can find some lapse points. Here in figure (1), one can see three curves representing $e^{-\lambda(r)}$ : the blue one indicating the curve for $\left(\alpha=0.1, T_{0}=-0.1\right)$, the maroon one denotes $\left(\alpha=0.5, T_{0}=-0.3\right)$ and finally the golden yellow denotes $\left(\alpha=1, T_{0}=-0.8\right)\left(D_{2}\right.$ has been taken as -0.2 in all cases). In all the three cases, we can see that our $g_{r r}$ meets a singularity.

\section{CONCLUSION}

In this paper, we have proposed a theoretical possibility of dark matter as an effect of $f(T)$ theory. Here, we don't have to consider the exotic matter (i.e. violates the usual energy condition ) hidden in the galactic halo. Employing certain restriction on the equation of state parameter of the luminous matter contained in the galactic region, we have shown flat rotation curve suggests the background geometry of the universe, in other words, spatial curvature of the universe can be obtained from a local gravitational phenomena. It is shown that the intrinsic property of the $f(T)$ inspired gravity is able to describe two crucial physical requirements - the stable circular orbits of the test particle out side of the halo and attractive gravity in the halo region. The approach used in this paper is totally different from other proposals exist in literature. We hope our model inspires the authors to give theoretical support of galactic dark matter and $\mathrm{f}(\mathrm{T})$ gravity is a matter of further investigation. 


\section{Acknowledgement :}

RB thanks ISRO grant "ISRO/RES/2/367/10-11" for providing Research Associate Fellowship. FR gratefully acknowledge support from IUCAA, Pune, India under Visiting Associateship under which a part of this work was carried out. FR is also thankful to PURSE, DST and UGC, Govt. of India for providing financial support.
[1] Zwicky F., 1933, Helv. Phys. Acta, 6, 110.

[2] F. Rahaman, M. Kalam, A. DeBenedictis, A. A. Usmani, and Saibal Ray, Mon. Not. R. Astron. Soc. 389, 27 (2008).

[3] K. K. Nandi, A.I. Filippov, F. Rahaman, Saibal Ray, A. A. Usmani, M. Kalam, and A. DeBenedictis, Mon. Not. R. Astron. Soc. 399, 2079 (2009).

[4] F. Rahaman et al, Phys.Lett. B694 , 10 (2010).

[5] T. Matos, F. S. Guzman, and D. Nunez, Phys. Rev. D 62, 061301 (2000).

[6] K.K. Nandi, I. Valitov, and N.G. Migranov, Phys. Rev. D 80, 047301 (2009).

[7] Matos T., Guzman F. S., Lopez L., 2000, Classical Quantum Gravity, 17, 1707.

[8] F. Rahaman et al, Int.J.Theor.Phys. 50 , 2655 (2011).

[9] F. Rahaman et al, Gen.Rel.Grav. 44 , 905 (2012).

[10] F. Rahaman et al, Phys. Lett. B714,131 (2012), e-Print: arXiv:1203.6649 gr-qc] .

[11] U. Nukamendi, M. Salgado, and D. Sudarsky, Phys. Rev. Lett., 84, 3037 (2000).

[12] T. Lee and B. Lee, Phys. Rev. D 69, 127502 (2004).

[13] F. Rahaman, R. Mondal, M. Kalam, and B. Raychaudhuri, Mod. Phys. Lett. A 22, 971 (2007).

[14] C. Moller, Mat. Fys. Skr. Dan. Vid. Selsk. 1 (1961) no. 10.

[15] C. Pellegrini and J. Plebanski, Mat. Fys. Skr. Dan. Vid. Selsk. 2 (1963) no. 4.; K. Hayashi and T. Shirafuji, Phys. Rev. D19 (1979) 3524

[16] R. Ferraro and F. Fiorini, Phys. Lett. B 702 (2011)
75 arXiv:1103.0824 [gr-qc]]; E. V. Linder, Phys. Rev. D 81, 127301 (2010) [Erratum-ibid. D 82, 109902 (2010)]; Puxun Wu and Hongwei Yu, Phys. Lett. B 693 415-420 (2010); Ratbay Myrzakulov, Eur. Phys. J. C 71, 1752 (2011); M. J. S. Houndjo, D. Momeni and R. Myrzakulov, arXiv:1206.3938v1 [physics.gen-ph]; M. Hamani Daouda, Manuel E. Rodrigues and $M$. J. S. Houndjo, arXiv:1205.0565v1 [gr-qc]; L. Baojiu, Thomas P. Sotiriou, John D. Barrow, Phys. Rev. D 83 104017 (2011); Shih-Hung Chen, J. B. Dent, S. Dutta and E. N. Saridakis, Phys. Rev. D 83023508 (2011); M.E. Rodrigues et al, Phys.Rev. D86 (2012) 104059, arXiv:1209.4859 [gr-qc].

[17] Böhmer et al, Class.Quant.Grav. 28 (2011) 245020.

[18] Hoyle, F., In problems of cosmical Aerodynamics, ed. J. M. Burgers and H. C. van de Hulst(Dayton : Central air Documents Office), 195 (1949).

[19] von Wiezsacker, C. F. , ApJ 114, 165(1951).

[20] Gamow, G. , Phys. Rev. 86, 251 (1952).

[21] Blumenthal, G. R., Faber, S. M., Primack, J. R., Rees, M. J. , Nature 311, 517 (1984).

[22] Barnes, J.E., Efstathiou, G., ApJ 319, 575(1987).

[23] V.C. DE Andrade et al, arXiv : gr-qc/0011087 1

[24] C. Deliduman, B. Yapiskan, arXiv:1103.2225 [gr-qc].

[25] Y.F. Cai et al, Class.Quan.Grav., 28, 215011 (2011).

[26] D. Lynden-Bell, J. Katz, and J. Bičák J, Phys. Rev. D 75, 024040 (2007). 